\title{
Philosophiques
}

\section{Les impasses du républicanisme de marché. À propos de Exit left, Markets and Mobility in Republican Thought, par Robert S. Taylor}

\section{Jean-Fabien Spitz}

Volume 44, numéro 2, automne 2017

URI : https://id.erudit.org/iderudit/1042344ar

DOI : https://doi.org/10.7202/1042344ar

Aller au sommaire du numéro

Éditeur(s)

Société de philosophie du Québec

ISSN

0316-2923 (imprimé)

1492-1391 (numérique)

Découvrir la revue

Citer cet article

Spitz, J.-F. (2017). Les impasses du républicanisme de marché. À propos de Exit left, Markets and Mobility in Republican Thought, par Robert S. Taylor.

Philosophiques, 44(2), 383-399. https://doi.org/10.7202/1042344ar d'utilisation que vous pouvez consulter en ligne. 


\title{
Étude critique
}

Les impasses du républicanisme de marché. À propos de Exit left, Markets and Mobility in Republican Thought, par Robert S. Taylor'

\author{
JEAN-FABIEN SPITZ \\ Département de philosophie \\ Université Paris 1 Panthéon Sorbonne
}

\section{Entre deux néo-républicanismes: la voie de la défection}

L'innovation majeure du républicanisme contemporain a consisté à récuser la définition de la liberté individuelle par l'absence d'obstacles ou d'interférences, et à la définir au contraire comme non-domination, c'est-à-dire comme l'absence de tout assujettissement effectif ou de toute vulnérabilité à la volonté arbitraire d'autrui. Robert Taylor partage cette intuition fondamentale et prétend donc s'inscrire dans le courant du républicanisme moderne. En revanche il se désolidarise du néo-républicanisme lorsqu'il s'agit des moyens à mettre en œuvre pour construire une société exempte de domination. Les néo-républicains ont en effet systématiquement proposé des solutions «politiques» à ce problème. Ils pensent qu'il convient de recourir à la loi et à l'État pour combattre les dominations privées qui naissent dans le monde de l'économie et de la société civile, et qu'il convient ensuite de recourir à d'autres moyens juridiques ou politiques - constitution, séparation des pouvoirs, rule of law, contrôle de constitutionnalité, procédures de contestation - pour empêcher que l'État ne devienne à son tour une source de domination sur les citoyens qu'il prétend protéger contre les dominations privées. P. Pettit lui-même, le principal représentant du courant néo-républicain, a clairement indiqué que cette seconde question quis custodiet custodes? - était au cœur d'une philosophie politique républicaine dont toute l'ambition consiste à construire un édifice institutionnel - une république - propre à contenir, par les voies de la loi et du droit, la domination que les citoyens pourraient exercer les uns sur les autres, tout en l'entourant de garde-fous et de procédures capables de l'empêcher de se muer lui-même en agent politique attaché à poursuivre ses propres intérêts aux dépens de ceux des citoyens ${ }^{2}$.

Le livre de Taylor est fondé sur l'idée que de tels moyens politiques et juridiques sont impuissants à juguler efficacement tant la domination privée

1. Robert S. Taylor, Exit left, Markets and Mobility in Republican Thought, Oxford, Oxford University Press, 2017, 144 pages.

2. P. Pettit, Républicanisme, une théorie de la liberté et du gouvernement, trad. fr. Paris, 2004; P. Pettit, On the People's Terms. A Republican Theory and Model of Democracy, Cambridge, 2012.

PHILOSOPHIQUES 44/2 - Automne 2017, p. 383-399 
que la domination publique qui menace d'être la conséquence de l'armement que la république veut donner à l'État pour contenir la domination privée. Non seulement ils sont impuissants mais ils sont dangereux, car ils consistent à construire un pouvoir - celui de la loi et de l'État - pour en combattre un autre et ainsi à déplacer le problème en l'accentuant au lieu de le résoudre ${ }^{3}$. Le pouvoir public destiné à combattre le pouvoir privé est en effet nécessairement plus puissant ou plus concentré que celui qu'il est censé tenir en lisière, et ses débordements ou abus, étant plus difficiles à contenir, exigent à leur tour des moyens de contrôle plus puissants et sophistiqués, dont l'abus est encore plus dangereux. En l'espèce, l'intuition de Taylor est que la mise en œuvre d'institutions de contrainte publique pour combattre des faits de contrainte privée aboutit à environner les individus d'une foule de pouvoirs qui, sans se faire réellement contrepoids, pèsent sur ses épaules et ne peuvent manquer de le dominer ${ }^{4}$. Dans la vie privée comme dans les relations entre producteurs et consommateurs, ou entre employeurs et employés, cette tentative de maîtrise directe, par des moyens juridiques et politiques, de la domination conduit à conférer à l'État et à ses agents des pouvoirs dont ils sont prêts à abuser. Même si ces pouvoirs ne sont pas discrétionnaires, ils comportent toujours une certaine latitude qui donne à la bureaucratie et aux fonctionnaires, une capacité de faire prévaloir leurs propres intérêts aux dépens de ceux des citoyens ${ }^{5}$.

Empruntant la distinction proposée par A. O. Hirschman entre défection et prise de parole, Taylor propose de classer l'ensemble des moyens politiques de contrôle de la domination dans la catégorie de la prise de parole et de s'écarter de l'analyse néo républicaine pour envisager des moyens de lutter contre la domination qui relèveraient au contraire de la défection ${ }^{6}$. Quiconque est menacé par un agent - public ou privé - qui tend à le dominer ou à lui imposer sa volonté, et à poursuivre ses propres intérêts à ses dépens, peut en effet recourir, selon Hirschman, à deux tactiques différentes: soit faire face, prendre la parole, élever la voix, contester, se grouper, construire des contre-pouvoirs (c'est ce que Hirschmann appelle «voice»), soit faire défection, s'en aller, échapper ainsi à l'emprise du dominateur potentiel, non pas en combattant ses entreprises mais en se soustrayant à son emprise, en se mettant hors de sa portée (ce que Hirschman appelle l'exit). En envisageant seulement des moyens politiques, juridiques et institutionnels, pour faire pièce à la domination publique et privée, les néo-républicains font confiance au voice. Taylor propose au contraire de

3. R. Taylor, Exit left, op. cit. p. 58.

4. R. Taylor, Exit Left, op. cit. p. 24. Voir F. Lovett, A General Theory of Domination and Justice (Oxford, 2010) pour l'idée qu'un État qui entreprendrait de maîtriser l'ensemble des dominations privées deviendrait à son tour une source inévitable de domination.

5. R. Taylor, Exit Left, op. cit. p. 9.

6. A. O. Hirschman, Défection et prise de parole, trad. fr. Fayard, Paris, 1995. 
faire confiance à des solutions économiques ${ }^{7}$. Le grand moyen de faire défection et d'échapper à la prise de la domination, selon Taylor, c'est en effet le marché: si un employeur tente de nous imposer des conditions indignes, le meilleur instrument dont nous puissions nous armer n'est pas de disposer de textes de loi qui nous confèrent le droit de faire condamner ses pratiques, mais d'avoir une possibilité effective de quitter notre emploi, doublée de dispositifs qui - à l'instar d'un revenu inconditionnel universel substantiel — permettent de se tenir de manière durable à l'écart du marché du travail et de la contrainte salariale ${ }^{8}$. Plus que tous les dispositifs juridiques issus de la prise de parole, c'est cette possibilité réelle que nous avons d'échapper à la prise du dominateur potentiel qui pourra discipliner ce dernier, le contraindre à avoir avec nous un mode d'échange équitable et non arbitraire. Il en va de même sur le marché des biens et des services: si un vendeur tente de faire prévaloir ses propres intérêts à nos dépens en pratiquant des prix indûment élevés ou en vendant des produits ou services de mauvaise qualité, la meilleure arme dont nous disposons contre lui est notre droit de porter notre clientèle ailleurs. C'est la perspective de notre défection qui le contraint à tenir compte de nos intérêts en matière de prix et de qualité car, si nous étions prisonniers sans pouvoir nous adresser ailleurs, il serait en mesure de nous imposer les conditions les plus désavantageuses en ignorant nos protestations les plus véhémentes. Sur un marché parfait où les consommateurs seraient entièrement informés et entièrement en mesure de se déplacer vers le mieux disant, aucun acteur ne pourrait en exploiter un autre, tous les vendeurs seraient obligés de prendre les prix tels qu'ils sont, c'est-à-dire tels qu'ils résultent de l'offre et de la demande, sans jamais pouvoir les manipuler, et aucun prestataire ne pourrait en rabattre sur la qualité de ce qu'il vend sans perdre aussitôt sa clientèle. Pour ce qui est des contrats de travail, tout salarié qui disposerait d'une possibilité effective de quitter son emploi sans avoir à supporter de coûts additionnels serait en mesure de "contraindre» son employeur à lui offrir des conditions équitables et une juste rémunération de son travail, et cela sans même avoir besoin d'exprimer ses revendications. L'exploitation deviendrait pour ainsi dire impossible, et la simple perspective de sa défection contraindrait là aussi l'employeur à proposer au salarié des conditions au moins aussi satisfaisantes que celles qu'il pourrait trouver ailleurs. Ces moyens indirects de parer à la domination comportent au demeurant l'immense avantage de court-circuiter et de rendre inutiles les dispositions réglementaires — en matière d'emploi, de

7. R. Taylor, "Market Freedom as Antipower», American Political Science Review, vol. $107 \mathrm{n}^{\circ}$ 3, 2013, p. 593-601.

8. Outre le revenu universel, Taylor mentionne aussi, parmi les moyens consistant à faciliter l'exit, la formation professionnelle et les demogrants ou aides à la création d'entreprises sous la forme de crédits garantis. 
prix, de salaires, de qualité - dont l'application confère inévitablement un pouvoir arbitraire à ceux qui sont chargés de les faire respecter.

Le républicanisme de marché ne nie cependant pas l'importance de la prise de parole sur le plan politique, car il a conscience que la possibilité de l'exit se restreint à mesure que l'on s'élève dans les niveaux auxquels s'exerce l'autorité, et que la possibilité de sortir d'un État dont on est membre sans encourir des coûts considérables est bien moindre que dans le contexte de la confrontation, par exemple, entre époux, entre vendeurs et consommateurs, ou entre employeurs et salariés. Il souligne en outre que les moyens d'un exit aisément accessible - en particulier un robuste revenu inconditionnel de base - ne peuvent être mis en place que par l'exercice de la parole démocratique au niveau de l'État, et il affirme donc que seul un exercice politique appuyé sur le voice peut garantir l'existence des conditions qui permettent l'efficacité du modèle économique. Il faut en effet une volonté politique pour que les conditions de l'exit soient réunies et que les individus disposent d'une possibilité effective de voter avec leurs pieds. Taylor prétend donc que son ambition n'est pas de réduire la liberté politique à la liberté du marché, mais de montrer que la réduction de la domination passe par la recherche d'un mixte entre plusieurs mécanismes de soumission du pouvoir aux préférences des citoyens, et que parmi ces mécanismes, la possibilité de sortir demeure un levier puissant, dont l'efficacité peut être supérieure à celle des moyens de contrôle politique et dont les inconvénients sont moindres. Il qualifie donc sa propre approche en disant qu'il s'agit d'un interventionnisme étatique orienté vers la défection, qui consiste à mettre les moyens politiques de la démocratie au service de robustes dispositifs de mobilité. Il montre aussi que ce mixte est variable selon le niveau de pouvoir auquel on se trouve, et que, plus on descend vers les pouvoirs ou organisations locaux, plus la possibilité de l'exit doit être employée pour maintenir ces pouvoirs en accord avec les intérêts des citoyens. Inversement, plus on remonte vers les pouvoirs nationaux, plus les moyens politiques de contrôle jouent un rôle important aux dépens de l'exit. Mais il soutient sans hésitation que, aux niveaux inférieurs d'exercice de l'autorité, et en particulier partout où pourrait s'exercer une domination privée - du mari sur son épouse, du vendeur sur le consommateur, de l'employeur sur ses salariés - l'accès à un exit facile et peu onéreux peut être mis en place et représente un moyen de lutte plus efficace que toutes les formes politiques ou juridiques de la prise de parole. En ce sens, les intérêts des consommateurs et des employés n'ont pas besoin d'être articulés ni débattus pour être identifiés et pris en considération par les dominateurs potentiels. La simple possibilité de transporter ailleurs leur clientèle ou leur travail contraint en effet ces derniers à tenir compte de leurs aspirations soit en ce qui concerne de qualité et de prix soit pour ce qui est des salaires et des conditions d'emploi. Il suffit au vendeur ou à l'employeur d'identifier les menaces ou les comportements de défection pour connaître les intérêts des consommateurs ou des salariés, et s'y 
conformer. C'est la possibilité même de l'exit qui les y contraint sans que celui-ci ait même besoin d'être mis en œuvre.

Il en va de même dans les rapports à l'intérieur du couple où, selon Taylor, la facilité du divorce et l'égalité des deux partenaires dans la capacité effective d'y avoir recours auraient pour conséquence de purger les relations entre conjoints de toute domination sans soumettre cette relation privée au regard et à l'action inquisitrice de la loi. Même sur le plan politique, Taylor entretient comme on va le voir un étrange fantasme où l'exercice du pouvoir pour la satisfaction des intérêts des citoyens serait maximisé par la facilité de ces derniers à se déplacer dans l'espace et à faire leur marché pour trouver les systèmes institutionnels et juridiques qui leur offrent le meilleur rapport qualité/prix, c'est-à-dire le meilleur équilibre entre les impôts qu'ils acquittent et les services collectifs dont ils bénéficient.

Ce qui manque au néo-républicanisme contemporain, dit Taylor, c'est donc d'une part une conscience plus aiguë du fait que la mise en œuvre de moyens politiques et juridiques pour combattre la domination privée comporte le risque inhérent d'engendrer une domination publique par l'État et par ses agents, et d'autre part la conscience qu'il existe d'autres moyens exempts des risques inhérents aux moyens politiques - de faire échec à cette domination privée, de discipliner ceux qui aspirent à l'exercer, et de les contraindre à s'en abstenir. Le marché serait ainsi un instrument de réalisation des valeurs républicaines, un moyen de faire que les individus ne sont jamais assujettis à la volonté arbitraire d'une autre personne comme ils le sont lorsqu'ils ne peuvent pas s'adresser à un autre employeur ou à un autre prestataire de services ou de biens. C'est le modèle économique du républicanisme, défini par un accord avec l'objectif républicain - la non-domination - mais aussi par une divergence essentielle sur les moyens de l'atteindre: pas exclusivement par des institutions républicaines, sur le danger desquelles il faut réfléchir, mais aussi et parfois essentiellement par les moyens non institutionnels du marché et de la concurrence. Pour protéger les plus vulnérables des entreprises dominatrices des tiers, la meilleure stratégie n'est pas toujours de les barder de droits et d'ériger autour d'eux les obstacles de la loi qui interdit certaines pratiques, mais de leur rendre l'exit possible, de leur donner un pouvoir de choisir'

\section{Les objections}

L'approche de Taylor se heurte cependant à de très fortes objections. Nous voudrions en évoquer ici essentiellement trois. Tout d'abord, l'idée que l'exit pourrait se substituer à la prise de parole nous semble reposer sur une méconnaissance de la complexité des rapports entre les deux options qui a pourtant été analysée en détail par Hirschman. Même aux niveaux inférieurs 
à l’État, comme le montre Hirschman, la meilleure stratégie pour empêcher le dysfonctionnement des organisations et des institutions n'est pas de privilégier exclusivement l'exit ou le voice, mais de pratiquer un délicat mélange entre les deux. L'approche de Taylor repose en second lieu sur une conception purement instrumentale de la politique qui est incohérente et en méconnaît les caractères principaux. Enfin, cette thèse ignore la distinction, pourtant capitale dans la problématique néo-républicaine, entre un pouvoir arbitraire et un pouvoir non arbitraire.

\section{a) L'exit: complément ou substitut du voice?}

Selon Taylor, l'exit est moins une pratique qu'une liberté, une porte ouverte qui, parce qu'elle existe, contraint celui à qui il est loisible d'échapper à mieux traiter les intérêts de ceux qui dépendent de lui et à mieux en tenir compte. C'est la possibilité de se soustraire à la domination qui contraint cette dernière, et non pas la réalité de la défection, dont la virtualité seule suffit à discipliner le pouvoir. Tel semble être le fil conducteur de sa démarche qui serait ainsi plus une étude des moyens de rendre la prise de parole effective que de la dévaloriser pour lui substituer la défection.

Cependant, lorsqu'il analyse en détail l'interaction entre les deux options, Taylor manifeste cependant une hésitation majeure et laisse ouvertement transparaître son peu de considération pour la prise de parole et sa volonté non pas de la renforcer, mais de la remplacer par la défection comme moyen de lutte contre la domination ${ }^{10}$.

À certains moments, Taylor semble pourtant bien affirmer que le principal avantage d'un exit aisé est de donner du poids à la prise de parole, de la renforcer, de contraindre le dominateur potentiel à écouter et à tenir compte des revendications de ses partenaires sous peine de les voir s'évanouir et cesser toute relation avec lui. Taylor suggère en ce sens que le voice sans l'exit est impuissant et que toute approche consistant à considérer que la fermeture des issues favorise la prise de parole en contraignant les citoyens, les consommateurs ou les salariés à y recourir, est une impasse. Ceux qui parlent n'ont de chance d'être écoutés que s'ils n'ont pas le dos au mur et si, en l'absence de réponse positive, ils peuvent opter pour la sortie. De même l'idée que le voice est la voie que doivent privilégier ceux qui ne disposent pas d'option réelle de sortie parce que la domination à laquelle ils sont assujettis est systémique est également une impasse puisque, si toutes les issues débouchent sur des relations aussi dominatrices que celles à laquelle on pré-

10. Taylor le dit explicitement: "Given the right circumstances (a broad array of providers with diverse characteristics and services) free choice and exit can act as effective substitutes for voice, revealed preferences as effective substitutes for stated preferences in compelling interferers to track the interests of interferees» (Exit Left, op. cit. p. 3). 
tend échapper, la prise de parole ne peut avoir aucune efficacité ${ }^{11}$. En d'autres termes, Taylor suggère à plusieurs reprises que l'exit ne se substitue pas à la prise de parole, à l'effort articulé pour faire que nos intérêts soient pris en considération et respectés par ceux qui sont en mesure d'exercer une autorité sur nous, mais qu'il lui donne de la consistance, du poids.

Hirschman lui-même soulignait cependant que, tout au contraire, la possibilité de l'exit avait pour effet d'affaiblir le voice et que ce dernier était d'autant plus puissant que l'ensemble des personnes potentiellement dominées étaient amenées à y avoir recours pour limiter ou contrôler cette domination. Quand il existe une possibilité de sortie, ceux qui sont le plus capables d'articuler les doléances et de mener la contestation ont tendance à en profiter et à faire défection, d'autant que ce sont aussi ceux qui ont le plus de capacité à en supporter le coût ${ }^{12}$.

Hirschman remarque en outre que les économistes occidentaux comme Taylor lui-même - ont du mal à admettre que les dispositifs les plus ouverts ne sont pas nécessairement les plus efficaces, et que la meilleure manière d'obtenir qu'une organisation serve au mieux les intérêts de ses utilisateurs ou de ses clients n'est pas nécessairement de donner à l'ensemble de ces derniers la possibilité de voter avec leurs pieds et d'aller ailleurs. Dans certains cas, dit-il «le monopole strict est préférable à une structure de marché moins rigide où la concurrence a sa place», et où «les structures closes sont supérieures aux structures ouvertes» parce que, dans ces dernières, la trop grande facilité de la défection interdit aux usagers de se tourner vers la structure elle-même pour en obtenir le redressement et en assurer le bon fonctionnement ${ }^{13}$. La défection, dit Hirschman, est en ce sens "loin de jouer son rôle d'instrument de redressement", car elle ne fait que "priver la firme ou l'organisation considérée de ses clients ou de ses adhérents les mieux qualifiés pour agir utilement de l'intérieur " alors même que la prise de parole serait susceptible d'aboutir à des résultats positifs ${ }^{14}$.

À cette objection, Taylor répond que l'exit ne fait obstacle au voice que lorsqu'il n'est accessible qu'à la minorité la plus capable de prendre la parole pour diagnostiquer et critiquer les dysfonctionnements et les points sur lesquels une institution cesse de servir les intérêts de ses usagers. En revanche, lorsqu'il devient aisément accessible à tous, il serait en mesure de le favoriser au lieu de l'entraver. L'idée est donc que la maîtrise de la domination passe par l'accroissement des moyens d'un exit effectif (une meilleure

11. Voir A. Gourevitch, "Labor Republicanism and the Transformation of Work", Political Theory, vol. $41 \mathrm{n}^{\circ} 4$ (2013), p. 591-617, pour le concept de domination systémique et l'idée que seul le recours au voice peut la réduire.

12. Hirschman, Défection et prise de parole, p. 80 et $86 ; 98-99 ; 122$.

13. Hirschman, ibid. Voir aussi R. Dagger «Neo-Republicanism and the Civic Economy ", Politics, Philosophy, and Economics, vol. 5, n 2 (2006), p. 151-173.

14. Hirschman, Défection et prise de parole, p. 92; ibid., p. 101, où Hirschman note que le Japon par exemple a tiré avantage du fait qu'il constitue une entité politique close. 
information, un revenu de base, etc.). C'est ce que Taylor appelle encourager indirectement le voice en facilitant l'exit (solution qui lui paraît préférable à celle qui consiste à encourager directement la prise de parole par des moyens législatifs, des droits conférés aux salariés, aux consommateurs, etc.). Par exemple, pour ce qui est du divorce, on prétend que la possibilité de l'exit a déstructuré les couples et les communautés, exposé les femmes à être abandonnées à leurs propres ressources, etc. Ce n'est vrai que lorsque le divorce est difficile et accessible seulement à ceux qui en ont les moyens, et que l'inégalité subsiste sur ce plan entre hommes et femmes. Quand, en revanche, le divorce est facile et également accessible pour les deux partenaires, on divorce moins, parce que les femmes arrivent à se faire entendre et obligent leurs conjoints à tenir compte de leurs revendications. C'est la thèse selon laquelle la possibilité de l'exit renforce le voice.

Mais Hirschman avait par avance fait bon marché de cette illusion en montrant que, si l'exit est accessible sans aucun coût, jamais la prise de parole ne se produit, soulignant ainsi l'ambiguité du rapport entre défection et prise de parole, et la difficulté de les associer l'une à l'autre pour combattre les différentes formes de domination. L'absence de toute possibilité de sortie dévalorise certes la parole et la rend inefficace, mais les portes trop largement ouvertes lui nuisent tout autant, et elles provoqueraient bel et bien une extinction de la prise de parole au profit d'une défection généralisée par laquelle les citoyens, au lieu de tenter de corriger les institutions et de les maintenir en conformité avec leurs intérêts, se dirigent vers celles qui leur offrent, à première vue et dans l'immédiat, des conditions plus avantageuses. Le facteur susceptible de renforcer l'efficacité de la prise de parole n'est donc pas la facilité de l'exit mais la nature du rapport que les individus entretiennent avec leurs institutions ou organisations sociales. Les institutions les mieux gouvernées - celles qui répondent le mieux aux intérêts de ceux qui les utilisent - ne sont ni totalement fermées ni totalement ouvertes, mais elles sont composées d'individus loyaux qui accordent de la valeur à ces institutions et aspirent à les maintenir en bon état de marche. Contrairement à l'attitude consumériste que Taylor veut promouvoir en imposant partout le modèle du marché, ces individus loyaux ne sont pas prêts à quitter le navire sitôt qu'il donne des signes de dysfonctionnement, et ils savent que l'exit manifeste un manque d'engagement et un manque de préoccupation pour la qualité des organisations et des relations entre les acteurs sociaux. Hirschman pense par conséquent que ce sont les organisations dont les membres font preuve d'une vigilance non consumériste et disposée à l'effort qui sont le mieux en mesure de combiner exit et voice. Si les organisations closes par autorité et dont les membres sont prisonniers rendent la parole inutile, les organisations totalement ouvertes où l'exit est absolument libre et aisé sont quant à elles incapables de susciter la prise de parole. La facilité de la sortie diminue l'attachement des membres pour leur organisation, elle décourage les efforts qu'il leur faudrait consentir pour prendre la parole, elle 
affaiblit leur vigilance et leur volonté de veiller à faire fonctionner les institutions pour l'intérêt général, elle encourage une sorte d'indifférence alimentée par le sentiment que, si les choses ne vont pas bien, c'est-à-dire si nos préférences explicites ne sont pas satisfaites, il est toujours possible d'aller ailleurs, et qu'il n'est donc pas indispensable de réfléchir aux moyens de redresser ou de corriger les errements des institutions auxquelles on a affaire ${ }^{15}$. Une telle facilité engendre donc une passivité qui interdit aux membres de s'investir activement pour maintenir le fonctionnement de l'organisation en conformité avec l'intérêt général. Mais lorsque l'organisation est telle que les membres sont loyaux, ils veulent la réformer plutôt que la quitter, et s'ils ont certes la possibilité de partir, ils ont aussi une réticence à le faire et préfèrent prendre la parole pour réformer les dysfonctionnements éventuels. Le loyalisme rend ainsi l'appartenance relativement rigide; tout en différant profondément d'une structure close, il freine la défection, même si celle-ci doit demeurer possible, et même si cette possibilité de dernier recours contribue à donner du poids à la parole du loyaliste ${ }^{16}$. Il est donc vrai que l'une des meilleures manières de faire pression sur une organisation est de la menacer de faire défection pour passer à une organisation rivale, et qu'une telle menace n'est crédible que s'il existe une telle organisation rivale, et s'il est effectivement possible de faire défection pour la rejoindre. Mais si l'absence complète de possibilité d'exit handicape la prise de parole, sa trop grande aisance l'entrave tout autant, car elle conduirait, face à des institutions, les citoyens à se comporter comme en présence d'entreprises substituables les unes aux autres et auxquelles ils ne sont pas particulièrement attachés: ils les laisseraient mourir de leur belle mort en ralliant d'autres institutions et en gagnant d'autres rivages.

L'analyse d'Hirschman ne conduit donc pas à faire de la facilité de l'exit le meilleur moyen de discipliner les dominateurs potentiels. Au contraire, ce genre d'effet redresseur sera maximalement obtenu par un mixte de clôture relative et de loyauté. À l'inverse, l'accent mis sur la facilitation de l'exit conduit donc Taylor, et c'est là que son hésitation se manifeste, à avaliser pleinement cette tactique de substitution qui consiste non pas à analyser les manques d'une institution, mais à la quitter quand elle ne nous satisfait plus. Il opte donc, dans les faits, non pour une tactique de consolidation de la prise de parole, mais pour une tactique qui vise à montrer que celle-ci, qui est à la fois coûteuse et dangereuse, peut être avantageusement remplacée par l'exit, aussi bien dans le domaine de la famille et de l'entreprise que dans le domaine politique. Il caresse en réalité l'idée que l'exit - s'il est rendu indolore et très peu coûteux — rendrait le voice superflu, et cela pour le plus grand avantage des dominés puisque la prise de parole et les moyens institutionnels qui lui sont associés sont à la fois oné-

15. R. Dagger, loc. cit.

16. Hirschman, op. cit. p. 131. 
reux et potentiellement créateurs de plus de domination qu'ils ne sont en mesure d'en guérir. La facilitation de l'exit aurait pour vertu essentielle de rendre superflus des mécanismes institutionnels de prise de parole qui ne font que reproduire et approfondir les dominations contre lesquelles elles prétendent lutter. On aboutirait ainsi à l'idée que l'exit rendrait la prise de parole inutile, qu'il se substituerait à elle parce que, dans un espace où la fluidité serait totale et les déplacements instantanés, il ne serait plus jamais nécessaire de protester contre un abus, une violation de droit, une attitude dominatrice. La simple manifestation de phénomènes de ce genre provoquant immédiatement le déplacement de ceux qui en sont l'objet vers des cieux plus cléments, les détenteurs de l'autorité sont amenés à s'autocontrôler en permanence pour éviter cette défection ${ }^{17}$.

\section{b) Une conception purement instrumentale de la politique}

La théorie républicaine contemporaine accorde une énorme importance à la prise de parole et aux moyens de la démocratie au niveau des entités politiques habilitées à définir les règles du jeu collectif. C'est au citoyen luimême à définir ses propres intérêts, à entrer en dialogue avec les détenteurs de l'autorité pour les lui faire connaître, par son vote, par la presse, par des manifestations, par la grève, à les rappeler à l'ordre en les écartant du pouvoir s'ils les méconnaissent, à mettre en œuvre des moyens de contestation pendant l'exercice des mandats, etc. Dans une telle approche, il est impossible que les intérêts des citoyens ne soient pas méprisés si ceux-ci ne les expriment pas et ne les défendent pas par des moyens politiques. Non seulement cette articulation leur est nécessaire pour faire connaître leurs intérêts aux gouvernants, mais elle est aussi nécessaire pour les identifier, car ce n'est que dans le contexte d'un débat que les citoyens peuvent, par la confrontation des opinions, dégager une vision de ce que sont leurs intérêts à long terme et de la manière de les concilier avec ceux de leurs concitoyens. La prise de parole dans un contexte démocratique, en ce sens, n'est pas un simple moyen de faire que les préférences des citoyens soient maximalement satisfaites, mais un outil d'interrogation de ces mêmes préférences pour déterminer la mesure dans laquelle elles constituent des intérêts. Il ne s'agit pas d'un moyen dont il faudrait seulement évaluer l'efficacité pour lui en substituer éventuellement d'autres, susceptibles de produire les mêmes effets. La prise de parole démocratique est une forme non substituable de contrôle d'institutions et d'organisations dont le dysfonctionnement n'implique pas la disparition, comme si toute institution était appelée à disparaître par désaffection aussitôt que ses "usagers » cessent d'en être satisfaits et ont la possibilité de porter leur clientèle ailleurs. Lorsqu'une organisation ou une institution d'une certaine ampleur, avec des ramifications et des

17. R. Taylor, Exit Left, op. cit. p. 3. 
connexions étendues, perd sa capacité à servir les intérêts de ceux qui l'utilisent, elle ne disparaît pas, elle survit comme un cancer qui affecte les tissus voisins et la vie sociale dans son ensemble. Le darwinisme sauvage l'adaptation ou la disparition - n'est pas la règle en matière sociale. La politique, en ce sens, a besoin d'instruments de correction, de guérison, pas d'élimination.

Le républicanisme de marché méconnaît donc le fait que la politique n'est pas seulement un outil de défense des préférences spontanées, mais aussi et surtout un dispositif d'action commune destiné à identifier des intérêts, à dégager ce qui pourrait être un intérêt commun, à formuler des règles de coexistence, et donc à identifier les conditions d'une non-domination qui ne peut pas se définir par le fait qu'un système social satisfait maximalement les préférences spontanées des citoyens. L'importance que Taylor continue d'accorder à la prise de parole dans le contexte d'une politique nationale tient par conséquent non pas à la nature même de la décision politique, à la nécessité de l'articulation et de la confrontation des opinions pour dégager ce qui pourrait relever d'un intérêt commun qui aurait besoin d'être défendu pour que le pouvoir ne revête pas le caractère de l'arbitraire, mais à la simple absence matérielle d'un monde aux frontières totalement effacées où le coût du déplacement d'une entité politique à une autre serait négligeable. Si les coûts de sortie des États constitués étaient voisins de zéro, dit Taylor, les citoyens et les entreprises pourraient faire leur marché entre différentes offres politiques comme les consommateurs font le leur entre différentes offres de machines à laver, et comme les salariés pourraient faire le leur entre différents employeurs si, là aussi, les coûts de sortie étaient nuls ou quasiment nuls. Dans cette approche, les intérêts des citoyens sont confondus avec leurs préférences non raisonnées et l'idée même d'intérêt commun, de règles de coopération qui soient équitables et donc acceptables par tous, a entièrement disparu ${ }^{18}$. De même, les institutions et les organisations sociales sont ici conçues comme des organismes vivants qui seraient, soit conduits à disparaître lorsque leur capacité à s'adapter à la demande de leurs clients s'étiole, soit au contraire capables de se réformer de l'intérieur sans avoir besoin que les raisons de leurs dysfonctionnements soient énoncées et portées sur la place publique. Dans les deux cas, le républicanisme marchand entretient le fantasme d'un monde sans discours politique, sans presse, sans opinion publique, sans investigation critique, sans mécanismes de décisions collectifs, un monde où les institutions et les organisations pourraient être disciplinées (condamnées à mort ou spontanément corrigées) par la simple addition des comportements individuels.

Cependant, cette approche méconnaît tout simplement qu'une société n'est pas une simple addition de comportements et de choix individuels. Les

18. Voir C. Sunstein, «Preferences and Politics », Philosophy and Public Affairs, vol. 20, $n^{\circ} 1,1991$, p. 3-34. 
institutions, par exemple, sont capables de survivre en état de dysfonctionnement - à la fois en raison de leur histoire et de leur pesanteur -, de susciter des attachements irrationnels et de développer des intérêts propres qui ne peuvent être mis au jour et disciplinés que par le discours et l'action collective. Le républicanisme marchand ignore ces phénomènes, et il n'admet la nécessité de la politique que parce que les comportements individuels ne sont ni parfaitement libres ni parfaitement informés.

Une telle approche ne tient aucun compte du caractère conflictuel des préférences. Certains citoyens vont exprimer une préférence pour des services publics de qualité et pour une taxation progressive des revenus, tandis que les entreprises vont aspirer à des charges sociales peu élevées, à un marché du travail flexible, à une taxation réduite de leurs profits. Toute société est dès lors contrainte d'arbitrer entre des intérêts opposés, et de déterminer collectivement les niveaux de fiscalité et de services publics qu'elle souhaite mettre en place. Mais Taylor ignore la nécessité de cette réflexion car, à ses yeux, la pratique politique possède une sorte d'objectif préformé sur lequel il ne peut exister aucun débat: elle doit maximiser dans tous les cas les possibilités de défection. Les États, dit-il, doivent mobiliser les moyens financiers et institutionnels indispensables pour garantir à tous les citoyens des moyens efficaces et non coûteux de se soustraire à toutes les relations qui risqueraient d'engendrer de la domination. Ils doivent donc s'employer à dissoudre les monopoles, à garantir que nul ne puisse faire les prix au lieu de se contenter de les accepter, et ils doivent surtout mettre en place un Revenu universel de base permettant à tous les salariés de se retirer au moins temporairement (mais pour quelle durée ?) du marché du travail sans avoir à en supporter les conséquences négatives en ce qui concerne leur revenu.

Pour le républicanisme de marché, cette maximisation des possibilités de défection serait aussi une maximisation de la satisfaction des préférences, laquelle serait confondue avec la prise en considération maximale des intérêts des citoyens. Mais cela ne tient pas compte du fait que nous avons besoin d'une décision politique pour ordonner les différentes options parce qu'il n'existe pas de hiérarchisation rationnelle unique des systèmes de satisfaction des préférences. La vision de la société politique comme outil de satisfaction de préférence spontanée semble ignorer complètement qu'il est impossible de hiérarchiser les systèmes en fonction de leur plus ou moins grande aptitude à satisfaire ces préférences prises de manière globale. Elle ignore donc qu'un ensemble d'institutions politiques est un outil de réflexion et d'arbitrage ou de choix entre des systèmes de satisfaction des préférences qui ne peuvent pas être disposés hiérarchiquement sur une seule ligne qui les rendrait commensurables ${ }^{19}$.

19. Voir J. Elster, "The Market and the Forum, Three Varieties of Political Theory». In Derek Matravers et Jonathan E. Pike (dir.), Debates in Contemporary Political Philosophy: An Anthology, Routledge, London, 2003. 
Taylor imagine enfin un système politique où chaque communauté un département par exemple ou même des unités plus réduites - doit payer les services qu'elle offre avec ses propres ressources. Dans ce système, que Taylor qualifie de "fédéralisme fiscal», chaque sous-unité (ville, département, province) aurait une autonomie fiscale presque complète, prenant elle-même les décisions sur le type de biens publics qu'elle veut offrir et à quel prix. Le gouvernement central ne serait plus en charge que des biens publics qui ne peuvent être que nationaux (la défense, l'air pur) et des dispositions qui garantissent à chacun les moyens de la mobilité. On accroîtrait ainsi considérablement, par rapport à un système qui s'efforce d'homogénéiser l'offre de services sur l'ensemble d'un territoire par des transferts politiquement ordonnés, le niveau de satisfaction des préférences individuelles et de bien être, chaque individu ayant en quelque sorte le niveau de biens publics qu'il préfère et pour lesquels il veut payer ${ }^{20}$.

Si certaines communautés sont pauvres et d'autres riches, la solution ne consiste donc pas à transférer nationalement de l'argent vers les premières, mais à faire en sorte que tous les individus aient les ressources adéquates pour se déplacer sans frais d'un point du territoire à l'autre. Les communautés qui offrent de mauvais services à des prix élevés seront désertées si elles ne réforment pas leurs pratiques, et elles seront puissamment incitées à les réformer dans le bon sens par la simple menace de perdre leurs administrés, et par conséquent leurs ressources. La centralisation fiscale et les transferts territoriaux ont au contraire des effets très pernicieux, car les exécutifs locaux qui dépendent des finances centrales de l'État, n'ont aucune incitation à améliorer la qualité de leurs propres services et à satisfaire leurs résidents parce que le vote par les pieds (le fait que les gens s'en vont) ne les affecte pas et que leurs ressources - allouées par l'État demeurent identiques.

Dans ce modèle, l'idée est que les mécanismes démocratiques (voice) sont superflus et même dangereux lorsqu'il s'agit de produire les meilleurs ensembles possibles de services aux citoyens. Il convient donc de leur substituer une concurrence parfaite entre régions et municipalités avec une possibilité réelle pour les citoyens de se déplacer de l'une à l'autre. On pourrait ainsi avoir une meilleure satisfaction des intérêts des citoyens sans les risques de la politique comme, par exemple, l'inféodation des dirigeants locaux ou provinciaux à des groupes d'intérêts ${ }^{21}$.

La politique comme marché, c'est-à-dire comme espace dans lequel les entreprises et les individus se déplacent à la recherche des partis, des institutions ou des législations les plus favorables à la satisfaction de leurs préférences spontanées, est bien l'horizon du républicanisme marchand, un horizon où le simple enregistrement des signaux envoyés par les prix et les

20. R. Taylor, Exit Left, op. cit., p. 78.

21. R. Taylor, Exit Left, op. cit. p. 72. 
comportements de défection tend à remplacer la parole politique articulée et à la rendre inutile. Pourquoi parler, en effet, pourquoi se doter d'institutions complexes de contestation et de prise de parole - surtout lorsqu'on sait à quel point elles sont potentiellement la source d'abus et d'exercices discrétionnaires du pouvoir - quand nous pouvons, par nos comportements, envoyer à nos partenaires des signaux matériels très explicites sur lesquels il ne leur est pas possible de se méprendre et qui les déterminent automatiquement à modifier leurs comportements dans le sens de nos intérêts s'ils ne veulent pas nuire aux leurs et perdre leur clientèle? À quoi bon élire des gouvernants si nous avons toujours le pouvoir d'aller ailleurs lorsque la manière dont ils nous gouvernent ne nous plaît pas? Le vote avec les pieds est donc le vote le plus efficace, celui qui ne risque pas d'être manipulé, alors que le processus démocratique est systématiquement exposé à des formes de corruption et ne garantit en rien que les gouvernants vont poursuivre les intérêts des citoyens au seul motif qu'ils sont élus et soumis à réélection. C'est ce fantasme d'une société où la parole politique serait devenue superflue, où nos préférences seraient manifestées par ce que nous faisons et non par ce que nous disons, qui domine l'approche de Robert Taylor ${ }^{22}$.

Comment un tel modèle pourrait-il fonctionner? Les phénomènes de ségrégation sociale vont inévitablement apparaître puisque les ménages aisés, peu demandeurs de services collectifs dont ils peuvent se dispenser, vont se diriger vers des communautés pratiquant une fiscalité réduite, laissant les citoyens les moins favorisés acquitter eux-mêmes, sans redistribution, les services publics dont ils ont besoin. Aucune facilité de déplacement ne peut remédier à cette ségrégation, car la venue de résidents pauvres exigeant des services publics substantiels et formant bientôt une majorité provoque la sécession des résidents riches, tout aussi mobiles, vers des cieux fiscalement plus cléments. De même, les tactiques pour attirer les entreprises et celles pour attirer les citoyens vont se révéler contradictoires car, pour attirer les premières, il faut baisser les impôts et compter sur les dépenses des cadres pour assurer le développement économique, alors que pour attirer les citoyens les plus pauvres, il faut au contraire des services publics et sociaux développés, et par conséquent coûteux.

Le fédéralisme fiscal provoquerait par conséquent les regroupements par classes sociales et aggraverait les inégalités. Il s'agit en réalité d'une théorie destinée à montrer qu'une société juste est seulement une société qui donne à chacun ce qu'il préfère et qui se passe de tout transfert, de toute

22. La théorie sociale établit aujourd'hui que les individus n'achètent pas nécessairement la quantité de services d'éducation et de santé dont ils ont objectivement besoin. Dans certains domaines on peut donc dire qu'il n'est pas possible de confondre préférences révélées et préférences réelles ni de prétendre que ce sont les arbitrages spontanés rendus par les individus qui maximisent leur bien être. Voir C. Sunstein \& R. Thaler, Nudge. Improving Decisions about Health, Wealth and Happiness, Penguin Books, 2009; C. Sunstein, Why Nudge? The Politics of Libertarian Paternalism, Princeton, 2014. 
redistribution et de toute solidarité. Elle semble en effet ignorer que les services publics sont quant à eux des mécanismes de transfert de ressources, parce que ceux qui les utilisent le plus sont aussi ceux qui paient le moins d'impôts tandis que ceux qui les utilisent le moins sont aussi ceux qui, en principe, paient le plus d'impôts. La mobilité parfaite des plus pauvres peut bien exister, mais elle ne saurait empêcher cette ségrégation sociale.

\section{c) Pouvoir public et pouvoir privé}

L'approche de Taylor repose enfin sur l'idée que les moyens publics mis en œuvre pour contenir les dominations privées engendrent ou risquent d'engendrer plus de domination, plus d'exposition à l'arbitraire, qu'ils n'en suppriment ${ }^{23}$. Mais cette idée nie l'une des thèses essentielles du néorépublicanisme, à savoir qu'il existe une différence essentielle entre un pouvoir public et un pouvoir privé. Le second est nécessairement arbitraire puisqu'il ne dépend que d'une volonté particulière, alors que le premier est assujetti à des règles formelles et substantielles, ainsi qu'à des procédures de contestation, qui sont en mesure de garantir son caractère non arbitraire.

Taylor calcule des quantités de domination en additionnant et en soustrayant des choses qui ne sont pas de même nature parce qu'on ne peut pas confondre une domination, c'est-à-dire une forme de pouvoir arbitraire et une autorité démocratique qui, par définition, parce qu'elle passe par la loi, est non arbitraire. La position de Taylor suppose en fait que cette différence ne peut pas exister, c'est-à-dire qu'il n'y a pas de différence entre une autorité privée et l'autorité d'une personne qui est mandatée par la loi et dont le pouvoir est strictement délimité par elle; il nie qu'il puisse exister un pouvoir non arbitraire, qui s'exerce dans l'intérêt de ceux qui lui sont assujettis, et il assimile purement et simplement pouvoir et pouvoir arbitraire ${ }^{24}$. Mais précisément toute la thèse républicaine repose sur la distinction entre le fait de subir une interférence arbitraire, et le fait de subir une interférence non arbitraire. L'idée que le pouvoir de la loi peut protéger contre la domination privée et créer des espaces protégés dans lesquels l'interférence des tiers est rendue impossible par la norme juridique échappe entièrement au républicanisme de marché.

Taylor ne conçoit donc la mise en œuvre des moyens politiques du voice que comme la construction d'un équilibre des pouvoirs, comme un jeu de forces, sans réfléchir sur la nature des pouvoirs impliqués et sur leur capa-

23. En réalité, Taylor nie tout simplement qu'il soit possible de dire que des dispositions publiques sont non arbitraires. Le pouvoir public et le pouvoir privé sont de même nature en réalité.

24. Pour illustrer l'idée que tout pouvoir de régulation implique une intervention arbitraire des tiers dans la vie des individus, Taylor cite le pouvoir dont disposait l'Église au Moyen Âge pour réguler les rapports matrimoniaux. Mais précisément, il n'existe aucune commune mesure entre de tels pouvoirs inquisitoriaux et une règle qui, par exemple, contraint le mari à obtenir la signature de son épouse pour un bien appartenant au couple. 
cité à être à la fois contrôlés et corrigés par des procédures démocratiques. Or le néo-républicanisme ne raisonne pas en fonction de l'équilibre des pouvoirs, et il n'entend pas conférer aux victimes potentielles de la domination des moyens de résistance, mais seulement édicter des règles qui interdisent publiquement la commission d'actes dominateurs et de conduites arbitraires. Il ne s'agit donc pas de combattre un pouvoir par un autre - stratégie qui comporterait effectivement les risques d'abus dénoncés par Taylor - mais de constitutionnaliser en quelque sorte le pouvoir privé en le soumettant à des règles dont la puissance publique est le garant.

La thèse du républicanisme marchand est qu'une telle constitutionnalisation est inutile parce que, lorsque la société est exclusivement constituée de contrats volontaires auxquels il est toujours possible de se soustraire, il nous est impossible d'entrer dans une relation à laquelle nous n'avons pas authentiquement consenti, et toutes les relations auxquelles nous avons explicitement et librement consenti sont exemptes de pouvoir puisque nous avons la capacité d'en sortir sitôt que nous le voulons. Le consommateur ne peut pas être dominé par le vendeur puisqu'il peut toujours choisir d'échapper aux conditions proposées par ce dernier; l'employé ne peut pas être dominé par l'employeur puisqu'il est toujours en mesure de démissionner tout en disposant - grâce au revenu inconditionnel — d'un autre moyen d'existence; le mariage ne peut donner lieu à la moindre domination puisque les conduites déviantes du conjoint sont soit automatiquement corrigées par la perspective de la défection de son épouse, soit rendues vaines et sans objet par la défection de celle-ci.

Cependant, cette démarche ne tient pas compte du fait que les sociétés développées ne sont pas des tissus de contrats privés. La production y est opérée par le biais de grandes entreprises dont les gains d'échelle sont assurés - par rapport aux petits producteurs - par le fait que précisément elles sont organisées hiérarchiquement et peuvent procéder par le biais du commandement et non du contrat. Comme le montre E. Anderson, les formidables progrès de la productivité du travail sont rendus possibles, dans le capitalisme moderne, non par la seule technologie, mais par une organisation du travail qui dispense les producteurs d'avoir à négocier de manière contractuelle l'ensemble des relations dont la production et ses variations sont l'occasion ${ }^{25}$. En ce sens, le pouvoir - en particulier à l'intérieur de l'entreprise - est inhérent au capitalisme moderne qui ne possède ses caractéristiques positives que parce qu'il s'agit d'un système qui est en mesure de substituer dans une large mesure le commandement au contrat et à la négociation entre partenaires égaux dans la sphère de la production. C'est précisément parce que ces formes de pouvoir existent, parce que l'asymétrie dans l'accès aux moyens de production permet de contraindre certains acteurs sociaux à s'enrôler au service des autres et à leur obéir, que les sociétés

25. E. Anderson, Private Government, op. cit. 
modernes ne peuvent garantir l'absence de domination en se contentant de garantir la liberté réelle des contrats et en garantissant à tous une sortie effective.

Taylor prétend que son approche est à la fois libertarienne - parce qu'elle fait prévaloir partout la liberté des échanges privés - et "de gauche » parce qu'elle assigne à l'État la mission de garantir cette liberté en offrant à tous les moyens d'un exit réellement accessible et non onéreux. Mais il ne paraît pas comprendre que cette supposée conciliation est une contradiction. La société contemporaine est fondée sur la possibilité de la contrainte, seule manière pour elle d'enrôler les hommes dans des organisations hiérarchiques qui sont efficaces parce qu'elles peuvent fonctionner autoritairement en substituant le commandement à la négociation et au contrat. C'est cette possibilité de contrainte qui fait son efficacité. Mais s'il en est ainsi, l'exit rendu accessible à tous sans le moindre coût signerait la fin de ce mode d'organisation de la production, car qui obéira s'il peut s'en dispenser? Ici, le fantasme libertarien d'une société où le contrat aurait éliminé la contrainte est pris à son propre piège. 